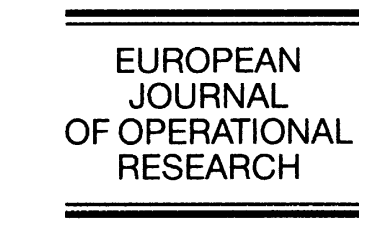

www.elsevier.com/locate/dsw

\title{
Some remarks on conflict analysis
}

\author{
Zdzisław Pawlak \\ Warsaw School of Information Technology, ul. Newelska 6, 01447 Warsaw, Poland
}

Received 1 September 2002; accepted 1 September 2003

Available online 12 August 2004

\begin{abstract}
Study of conflicts is of greatest importance both practically and theoretically. Conflict analysis and resolution play an important role in business, governmental, political and lawsuits disputes, labor-management negotiations, military operations and others. Many formal models of conflict situations have been proposed and studied. In this paper we outline a new approach to conflict analysis, which will be illustrated by a simple tutorial example of voting analysis in conflict situations.
\end{abstract}

(c) 2004 Elsevier B.V. All rights reserved.

Keywords: Conflict analysis; Rough sets; Decision analysis

\section{Introduction}

Conflict analysis and resolution play an important role in business, governmental, political and lawsuits disputes, labor-management negotiations, military operations and others. To this end many mathematical formal models of conflict situations have been proposed and studied, e.g. [1-6,9].

Various mathematical tools, e.g., graph theory, topology, differential equations and others, have been used to that purpose.

Needless to say that game theory can be also considered as a mathematical model of conflict situations.

E-mail address: zpw@ii.pw.edu.pl.
In fact there is no, as yet, "universal" theory of conflicts and mathematical models of conflict situations are strongly domain dependent.

We are going to present in this paper still another approach to conflict analysis, based on some ideas of rough set theory-along the lines proposed in [6]. We will illustrate the proposed approach by means of a simple tutorial example of voting analysis in conflict situations.

The considered model is simple enough for easy computer implementation and seems adequate for many real life applications but to this end more research is needed.

Last but not least, it is worthwhile to mention that conflict analysis seems to be important for 
decision theory. Rough set based decision support plays important role in decision theory, see e.g. [8], but in order to exploit this relationship in conflict analysis more research is needed.

\section{Basic concepts of conflict theory}

In this section we give after [6] definitions of basic concepts of the proposed approach.

Let us assume that we are given a finite, nonempty set $U$ called the universe. Elements of $U$ will be referred to as agents. Let a function $v: U \rightarrow\{-1,0,1\}$, or in short $\{-, 0,+\}$, be given assigning to every agent the number $-1,0$ or 1 , representing his opinion, view, voting result, etc. about some discussed issue, and meaning against, neutral and favorable, respectively.

In order to express relations between agents we define three basic binary relations on the universe: conflict, neutrality and alliance. To this end we first define the following auxiliary function:

$$
\phi_{v}(x, y)= \begin{cases}1 & \text { if } v(x) v(y)=1 \text { or } x=y, \\ 0 & \text { if } v(x) v(y)=0 \text { and } x \neq y, \\ -1 & \text { if } v(x) v(y)=-1\end{cases}
$$

This means that, if $\phi_{v}(x, y)=1$, agents $x$ and $y$ have the same opinion about issue $v$ (are allied on $v$ ); if $\phi_{v}(x, y)=0$ means that at least one agent $x$ or $y$ has neutral approach to issue $v$ (is neutral on $v$ ), and if $\phi_{v}(x, y)=-1$, means that both agents have different opinions about issue $v$ (are in conflict on $v$ ).

In what follows we will define three basic relations $R_{v}^{+}, R_{v}^{0}$ and $R_{v}^{-}$on $U^{2}$ called alliance, neutrality and conflict relations respectively, and defined as follows:

$$
\begin{aligned}
& R_{v}^{+}(x, y) \quad \text { iff } \phi_{v}(x, y)=1, \\
& R_{v}^{0}(x, y) \quad \text { iff } \phi_{v}(x, y)=0, \\
& R_{v}^{-}(x, y) \quad \text { iff } \phi_{v}(x, y)=-1 .
\end{aligned}
$$

It is easily seen that the alliance relation has the following properties:

(i) $R_{v}^{+}(x, x)$,

(ii) $R_{v}^{+}(x, y)$ implies $R_{v}^{+}(y, x)$,

(iii) $R_{v}^{+}(x, y)$ and $R_{v}^{+}(y, z)$ implies $R_{v}^{+}(x, z)$, i.e., $R_{v}^{+}$is an equivalence relation. Each equivalence class of alliance relation will be called coalition with respect to $v$. Let us note that the condition (iii) can be expressed as "a friend of my friend is my friend".

Remark. Formation of coalitions is a very import issue in conflict analysis and many results have been obtained in this area. However we refrain to discuss this problem here in detail, and we want only to point out that the idea of coalition is a consequence of the assumed alliance relation.

For the conflict relation we have the following properties:

(iv) $\operatorname{not} R_{v}^{-}(x, x)$,

(v) $R_{v}^{-}(x, y)$ implies $R_{v}^{-}(y, x)$,

(vi) $R_{v}^{-}(x, y)$ and $R_{v}^{-}(y, z)$ implies $R_{v}^{+}(x, z)$,

(vii) $R_{v}^{-}(x, y)$ and $R_{v}^{+}(y, z)$ implies $R_{v}^{-}(x, z)$.

Conditions (vi) and (vii) refer to well-known sayings "an enemy of my enemy is my friend" and "a friend of my enemy is my enemy".

For the neutrality relation we have

(viii) $\operatorname{not} R_{v}^{0}(x, x)$,

(ix) $R_{v}^{0}(x, y)=R_{v}^{0}(y, x)$.

Let us observe that in the conflict and neutrality relations there are no coalitions.

The following property holds $R_{v}^{+} \cup R_{v}^{0} \cup R_{v}^{-}=$ $U^{2}$ because if $(x, y) \in U^{2}$ then $\Phi_{v}(x, y)=1$ or $\Phi_{v}(x, y)=0$ or $\Phi_{v}(x, y)=-1$ so $(x, y) \in R_{v}^{+}$or $(x, y) \in R_{v}^{0}$ or $(x, y) \in R_{v}^{-}$. All the three relations $R_{v}^{+}, R_{v}^{0}, R_{v}^{-}$are pairwise disjoint, i.e., every pair of objects $(x, y)$ belongs to exactly one of the above defined relations (is in conflict, is allied or is neutral).

With every conflict situation we will associate a conflict graph $G=\left(R_{v}^{+}, R_{v}^{0}, R_{v}^{-}\right)$.

An example of a conflict graph is shown in Fig. 1.

Solid lines are denoting conflicts, dotted linesalliance, and neutrality, for simplicity, is not shown explicitly in the graph. Of course, B, C, and $\mathrm{D}$ form a coalition.

\section{Information systems and decision tables}

In what follows we will employ basic concepts of rough set theory to analysis of conflict situations. 


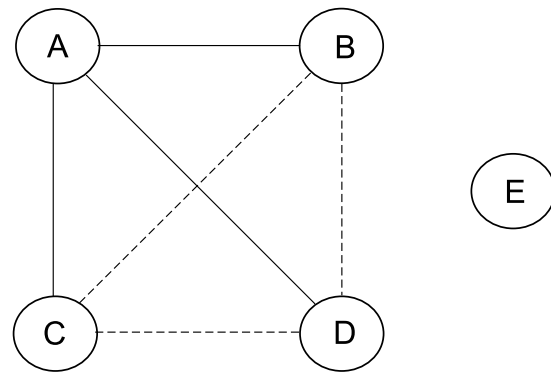

Fig. 1. Exemplary conflict graph.

An information system is a data table, whose columns are labeled by attributes, rows are labeled by objects of interest and entries of the table are attribute values.

Formally, by an information system we will understand a pair $S=(U, A)$, where $U$ and $A$, are finite, non-empty sets called the universe, and the set of attributes, respectively. With every attribute $a \in A$ we associate a set $V_{a}$, of its values, called the domain of $a$. Any subset $B$ of $A$ determines a binary relation $I(B)$ on $U$, which will be called an indiscernibility relation, and defined as follows: $(x, y) \in I(B)$ if and only if $a(x)=a(y)$ for every $a \in A$, where $a(x)$ denotes the value of attribute $a$ for element $x$. Obviously $I(B)$ is an equivalence relation. The family of all equivalence classes of $I(B)$, i.e., a partition determined by $B$, will be denoted by $U / I(B)$, or simply by $U / B$; an equivalence class of $I(B)$, i.e., block of the partition $U / B$, containing $x$ will be denoted by $B(x)$ and referred to as B-elementary set or B-granule induced by $x$.

If $(x, y)$ belongs to $I(B)$ we will say that $x$ and $y$ are $B$-indiscernible (indiscernible with respect to $B$ ).

If we distinguish in an information system two disjoint classes of attributes, called condition and decision attributes, respectively, then the system will be called a decision table and will be denoted by $S=(U, C, D)$, where $C$ and $D$ are disjoint sets of condition and decision attributes, respectively.

For the purpose of conflict analysis considered in this paper we assume that $U$ represents agents, condition attributes are meant to describe features of agents (e.g., sex, age, etc.) and the only decision attribute is $v$ with values $1,0,-1(+, 0,-)$ repre- senting opinion of agents. It is also possible to interpret the proposed model differently (see e.g. $[6])$.

\section{Decision rules and decision tables}

Every decision table describes decisions determined, when some conditions are satisfied. In other words each row of the decision table specifies a decision rule which determines decisions in terms of conditions.

Let us describe decision rules more exactly.

Let $S=(U, C, D)$ be a decision table. Every $x \in U$ determines a sequence $c_{1}(x), \ldots, c_{n}(x)$, $d_{1}(x), \ldots, d_{m}(x) \quad$ where $\quad\left\{c_{1}, \ldots, c_{n}\right\}=C \quad$ and $\left\{d_{1}, \ldots, d_{m}\right\}=D$.

The sequence will be called a decision rule induced by $x$ (in $S$ ) and denoted by $c_{1}(x), \ldots, c_{n}(x) \rightarrow d_{1}(x), \ldots, d_{m}(x)$, in short $C \rightarrow{ }_{x} D$ or $C \rightarrow D$ when $x$ is known.

The number $\operatorname{supp}_{x}(C, D)=|C(x) \cap D(x)|$ will be called the support of the decision rule $C \rightarrow{ }_{x} D$, where $C(x)$ and $D(x)$ denote $C$ and $D$ granules induced by $x$, respectively and $|X|$ denotes the cardinality of $X$.

The number

$\sigma_{x}(C, D)=\operatorname{supp}_{x}(C, D) /|U|$

will be referred to as the strength of the decision rule $C \rightarrow{ }_{x} D$.

With every decision rule $C \rightarrow{ }_{x} D$ we associate the certainty factor of the decision rule, denoted $\operatorname{cer}_{x}(C, D)$ and defined as follows:

$$
\begin{aligned}
\operatorname{cer}_{x}(C, D) & =\operatorname{supp}_{x}(C, D) /|C(x)| \\
& =\sigma_{x}(C, D) / \pi(C(x)),
\end{aligned}
$$

where $\pi(C(x))=|C(x)| /|U|$.

The certainty factor may be interpreted as conditional probability that $y$ belongs to $D(x)$ given $y$ belongs to $C(x)$, symbolically $\pi_{x}(D \mid C)$, or as certainty to which $C$ implies $D$.

If $\operatorname{cer}_{x}(C, D)=1$, then $C \rightarrow{ }_{x} D$ will be called a certain decision rule in $S$; if $0<\operatorname{cer}_{x}(C, D)<1$ the decision rule will be referred to as an uncertain decision rule in $S$. 
Besides, we will also use a coverage factor of the decision rule, denoted $\operatorname{cov}_{x}(C, D)$ and defined as

$$
\begin{aligned}
\operatorname{cov}_{x}(C, D) & =\operatorname{supp}_{x}(C, D) /|D(x)| \\
& =\sigma_{x}(C, D) / \pi(D(x)),
\end{aligned}
$$

where $\pi(D(x))=|D(x)| /|U|$.

Similarly, $\operatorname{cov}_{x}(C, D)=\pi_{x}(C \mid D)$.

If $C \rightarrow{ }_{x} D$ is a decision rule, then $D \rightarrow{ }_{x} C$ is the inverse decision rule. Inverse decision rules can be used to give explanations for decisions.

Let us observe that the coverage factor is the certainty factor for the inverse decision rule.

For many applications exact values of certainty of coverage factors of decision rules are not necessary. To this end we introduce "approximate" decision rules, denoted $C \Rightarrow D$ and read " $C$ mostly implies $D$ ". $C \Rightarrow D$ if and only if $\operatorname{cer}(C, D)>0.5$.

\section{Flow graphs and decision tables}

With every decision table we associate a flow graph, i.e., a directed, connected, acyclic graph defined as follows: to every decision rule $C \rightarrow{ }_{x} D$ we assign a directed branch $x$ connecting the input node $C(x)$ and the output node $D(x)$. Strength of the decision rule represents a throughflow of the corresponding branch. Thus branches of the flow graph connect $C$-granules and $D$-granules of the graph.

Flow distribution analysis can be used to study the relationship between agents involved in the conflict.

More about flow graphs can be found in [7].

\section{An example}

In this section we will illustrate the above presented ideas by means of a very simple tutorial example.

Table 1 presents a decision table in which the only condition attribute is Party, whereas the decision attribute is Voting. The table describes voting results in a parliament containing 500 members grouped in four political parties denoted $A, B, C$ and $D$. Suppose the parliament discussed certain
Table 1

Voting result

\begin{tabular}{llll}
\hline Fact & Party & Voting & Support \\
\hline 1 & $A$ & + & 200 \\
2 & $A$ & 0 & 30 \\
3 & $A$ & - & 10 \\
4 & $B$ & + & 15 \\
5 & $B$ & - & 25 \\
6 & $C$ & 0 & 20 \\
7 & $C$ & - & 40 \\
8 & $D$ & + & 25 \\
9 & $D$ & 0 & 35 \\
10 & $D$ & - & 100 \\
\hline
\end{tabular}

issue (e.g. membership of the country in European Union) and the voting result is presented in column Voting, where +, 0 and - denoted yes, abstention and no, respectively. The column support contains the number of voters for each option.

The strength, certainty and the coverage factors for Table 1 are given Table 2.

From the certainty factors we can conclude, for example, that

- $83.3 \%$ of party $A$ voted yes,

- $12.5 \%$ of party $A$ abstained,

- $4.2 \%$ of party $A$ voted no.

From the coverage factors we can get, for example, the following explanation of voting results:

- $83.3 \%$ yes votes came from party $A$.

- $6.3 \%$ yes votes came from party $B$.

- $10.4 \%$ yes votes came from party $C$.

Table 2

Certainty and coverage factors

\begin{tabular}{llll}
\hline Fact & Strength & Certainty & Coverage \\
\hline 1 & 0.40 & 0.833 & 0.833 \\
2 & 0.06 & 0.125 & 0.353 \\
3 & 0.02 & 0.042 & 0.057 \\
4 & 0.03 & 0.375 & 0.063 \\
5 & 0.05 & 0.625 & 0.143 \\
6 & 0.04 & 0.333 & 0.235 \\
7 & 0.08 & 0.667 & 0.229 \\
8 & 0.05 & 0.156 & 0.104 \\
9 & 0.07 & 0.219 & 0.412 \\
10 & 0.20 & 0.625 & 0.571 \\
\hline
\end{tabular}




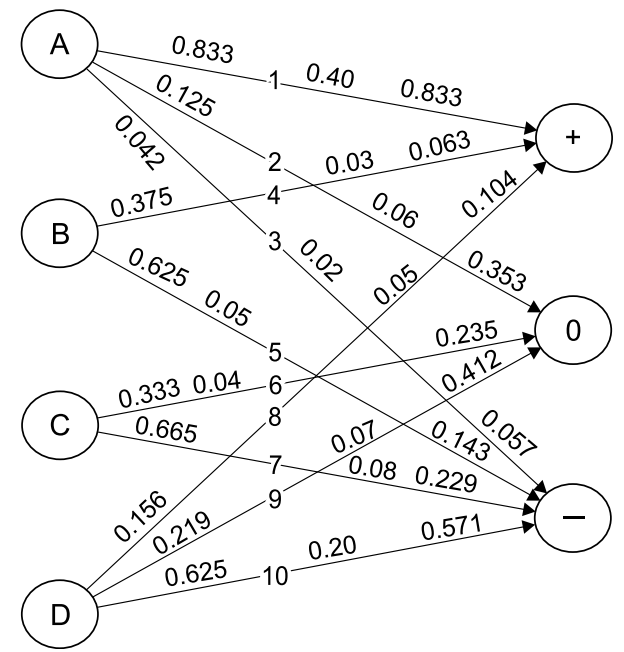

Fig. 2. Flow graph.

The flow graph associated with Table 2 is shown in Fig. 2.

Branches of the flow graph represent decision rules together with their certainty and coverage factors. For example, the decision rule $A \rightarrow 0$ has the certainty and coverage factors 0.125 and 0.353 , respectively.

The flow graph gives a clear insight into the voting structure of all parties.

We can replace flow graph shown in Fig. 2 by "approximate" flow graph presented in Fig. 3.

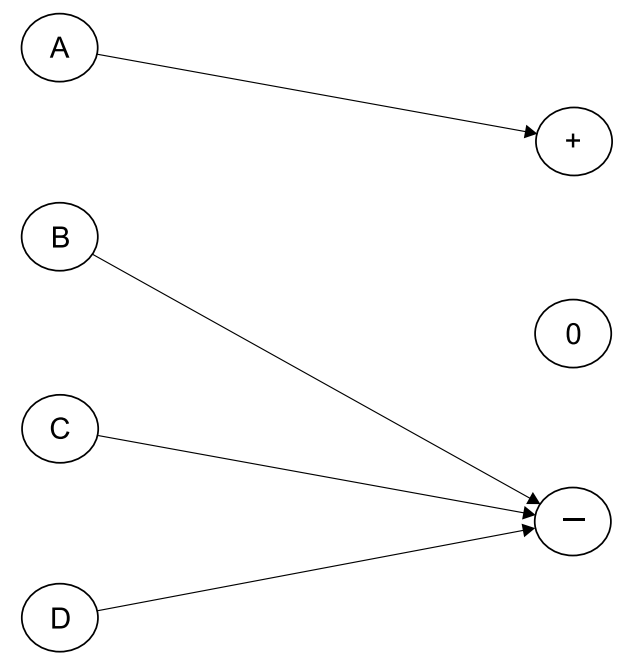

Fig. 3. Approximate flow graph.

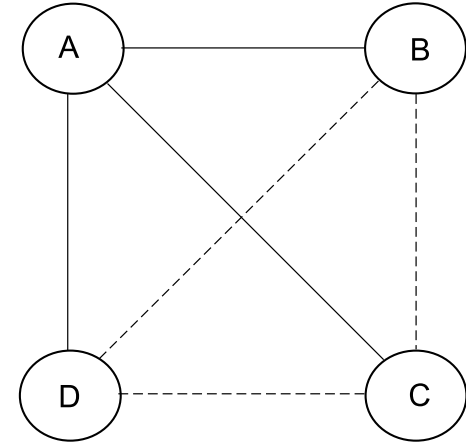

Fig. 4. Conflict graph.

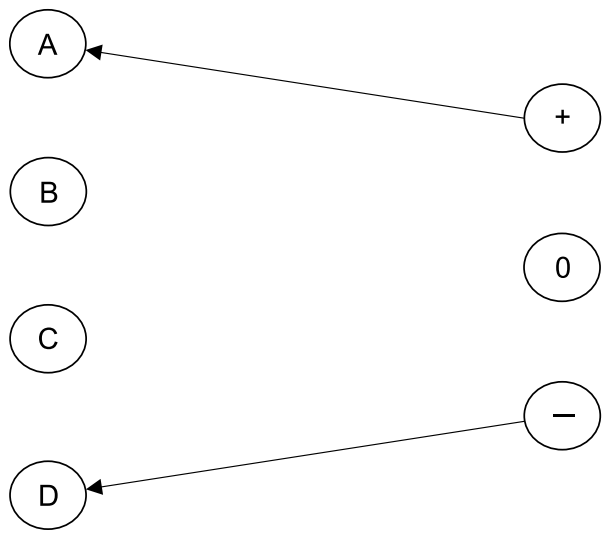

Fig. 5. Inverse approximate flow graph.

From this graph we can see that parties $B, C$ and $D$ form a coalition, which is in conflict with party $A$, i.e., every member of the coalition is in conflict with party $A$. The corresponding conflict graph is shown in Fig. 4. Moreover from the flow graph shown in Fig. 2 we can obtain an "inverse" approximate flow graph which is shown in Fig. 5.

This flow graph contains all inverse decision rules with certainty factor greater than 0.5 . From this graph we can see that yes votes were obtained mostly from party $A$ and no votes mostly from party $D$.

\section{Summary}

This paper contains a new approach to conflict analysis. The approach is based on some ideas of 
rough set theory. Moreover it is shown that with any conflict situation a flow graph can be associated. Flow distribution in the graph can be used to study the relationship between agents involved in the conflict.

\section{Acknowledgement}

Thanks are due to the anonymous referees as for their critical remarks.

\section{References}

[1] J.L. Casti, Alternative Realities-Mathematical Models of Nature and Man, Wiley, New York, 1989.

[2] C.H. Coombs, G.S. Avruin, The Structure of Conflicts, Lawrence Erlbaum, London, 1988.
[3] R. Deja, Conflict analysis, rough set methods and applications, in: L. Polkowski, S. Tsumoto, T.Y. Lin (Eds.), Studies in Fuzzyness and Soft Computing, Physica-Verlag, A Springer-Verlag Company, Wien, 2000, pp. 491-520.

[4] Y. Maeda, K. Senoo, H. Tanaka, Interval density function in conflict analysis, in: N. Zhong, A. Skowron, S. Ohsuga (Eds.), New Directions in Rough Sets, Data Mining and Granular-Soft Computing, Springer, New York, 1999, pp. 382-389.

[5] A. Nakamura, in: S.K. Pal, A. Skowron (Eds.), Conflict Logic with Degrees, Rough Fuzzy Hybridization-A New Trend in Decison-Making, Springer, New York, 1999, pp. 136-150.

[6] Z. Pawlak, An inquiry into anatomy of conflicts, Journal of Information Sciences 109 (1998) 65-68.

[7] Z. Pawlak, Rough sets, Bayes' theorem and flow graphs, in: IMPU 2002, 2002, pp. 1619-1624.

[8] R. Slowinski, Rough set approach to decision analysis, AI Expert 10 (1995) 18-25.

[9] F. Roberts, Discrete Mathematical Models with Applications to Social, Biological and Environmental Problems, Prince-Hall, Englewood Cliffs, NJ, 1976. 